Proceedings of the Edinburgh Mathematical Society (2006) 49, 29-37 (C)

DOI:10.1017/S0013091504000999 Printed in the United Kingdom

\title{
LEGENDRIAN GRAPHS GENERATED BY TANGENTIAL FAMILY GERMS
}

\author{
GIANMARCO CAPITANIO \\ Université Paris Dauphine, Place du Maréchal de Lattre de Tassigny, \\ 75775 Paris Cedex 16, France (capitani@ceremade.dauphine.fr)
}

(Received 21 September 2004)

\begin{abstract}
We construct a Legendrian version of envelope theory. A tangential family is a one-parameter family of rays emanating tangentially from a regular plane curve. The Legendrian graph of the family is the union of the Legendrian lifts of the family curves in the projectivized cotangent bundle $P T^{*} \mathbb{R}^{2}$. We study the singularities of Legendrian graphs and their stability under small tangential deformations. We also find normal forms of their projections into the plane. This allows us to interpret the beak-to-beak perestroika as the apparent contour of a deformation of the double Whitney umbrella singularity $A_{1}^{ \pm}$.
\end{abstract}

Keywords: envelope theory; contact geometry; tangential families; Legendrian graphs

2000 Mathematics subject classification: Primary 14B05; 53C15; 58K25

\section{Introduction}

A tangential family is a one-parameter family of rays (i.e. regular plane curves) emanating tangentially from a regular plane curve. Tangential families and their envelopes (or caustics) are natural objects in differential geometry: for instance, every curve in a Riemannian surface defines the tangential family of its tangent geodesics. The theory of tangential families is related to the study developed by Thom and Arnol'd for plane envelopes (see $[\mathbf{1}, \mathbf{2}, \mathbf{1 6}]$ ). In $[\mathbf{7}]$ and $[\mathbf{8}]$ we studied stable and simple singularities of tangential family germs with respect to deformations among tangential family germs and $\mathcal{A}$-equivalence.

In this paper we construct a Legendrian version of tangential family theory. The envelope of a tangential family is viewed as the apparent contour of the surface, called a Legendrian graph, formed by the union of the Legendrian lifts of the family curves in the projectivized cotangent bundle of the plane.

We classify the Legendrian graph singularities that are stable under small tangential deformations of the generating tangential families. We prove that, in addition to a regular Legendrian graph, there exists just one more local stable singularity, the double Whitney umbrella $A_{1}^{ \pm}$. Furthermore, we find normal forms of typical projections of Legendrian graphs into the plane. This allows us to interpret the beak-to-beak perestroika 
as the apparent contour of a non-tangential deformation of the double Whitney umbrella singularity in the projectivization of the cotangent bundle $T^{*} \mathbb{R}^{2}$.

Our results are related to several theories, concerning maps from the plane to the space $[\mathbf{1 4}, \mathbf{1 7}]$, projections of manifolds with boundaries $[\mathbf{5}, \mathbf{1 2}]$, singular Lagrangian varieties and their Lagrangian mappings $[\mathbf{1 0}]$, and the theory of Clairaut-type equations $[\mathbf{1 3}, \mathbf{1 5}]$.

\section{Legendrian graphs and their singularities}

Unless otherwise specified, all the objects considered below are supposed to be of class $\mathcal{C}^{\infty}$; by plane curve we mean a smooth map $\mathbb{R} \rightarrow \mathbb{R}^{2}$. This curve is said to be regular at every point at which its first derivative is not zero.

In this section we recall basic facts about tangential families and we define their Legendrian graphs in the projectivized cotangent bundle $P T^{*} \mathbb{R}^{2}$. We study the typical singularities of these graphs up to $\mathcal{A}$-equivalence. This classification considers neither the fibre nor the contact structure of $P T^{*} \mathbb{R}^{2}$. A classification of Legendrian graphs taking into account the fibre bundle structure is the object of $\S 3$.

Let $f: \mathbb{R}^{2} \rightarrow \mathbb{R}^{2}$ be a mapping of the source (fibred) plane $\mathbb{R}^{2}=\mathbb{R} \times \mathbb{R}$, equipped with the coordinates $\xi$ and $t$, to another plane. We set $f_{\xi}(t):=f(\xi, t)$.

Definition. The family of curves $\left\{f_{\xi}: \xi \in \mathbb{R}\right\}$ is a tangential family if $\xi \mapsto f(\xi, 0)$ is a regular curve, called a support, and for every $\xi \in \mathbb{R}$, the curve $f_{\xi}$ is everywhere regular and tangent to the support at $f(\xi, 0)$.

In particular, the partial derivatives $\partial_{\xi} f(\xi, 0)$ and $\partial_{t} f(\xi, 0)$ are parallel non-zero vectors for every $\xi \in \mathbb{R}$.

The graph of such a family is the surface $\Phi:=\left\{\left(\xi, f_{\xi}(t)\right): \xi, t \in \mathbb{R}\right\} \subset \mathbb{R}^{3}$. The envelope is the apparent contour of $\Phi$ under the projection $\pi: \mathbb{R}^{3} \rightarrow \mathbb{R}^{2}, \pi(\xi, P):=P$ (i.e. the critical value set of $\left.\left.\pi\right|_{\Phi}\right)$; the criminant set is the critical set of $\left.\pi\right|_{\Phi}$. By the very definition, the support of a tangential family belongs to its envelope.

Let $p$ be a non-negative integer. A $p$-parameter deformation $F: \mathbb{R}^{2} \times \mathbb{R}^{p} \rightarrow \mathbb{R}^{2}$ of a tangential family $f$ is tangential if $F_{\lambda}:=F(\cdot ; \lambda)$ is a tangential family for every $\lambda$. Note that the supports of the deformed families form a smooth deformation of the support of the initial family.

Below we will consider tangential family germs. Note that graphs of tangential family germs are germs of embedded surfaces. We will denote by $s$ and $t$ the variables of the source plane $\mathbb{R}^{2}$ when this space is not equipped with the fibration $\mathbb{R} \times \mathbb{R}$, leaving the variable $\xi$ for the distinguished parameter of a tangential family germ in the fibred case.

Remark. A tangential family germ may be $\mathcal{A}$-equivalent to a map germ which is not a tangential family germ. However, $\mathcal{A}$-equivalence preserves the major feature of the families of curves, namely, their envelopes. In [7] we proved that there are exactly two tangential family singularities which are $\mathcal{A}$-stable under small tangential deformations. These singularities, denoted by I and II, are represented, respectively, by the tangential family germs $\left(\xi+t, t^{2}\right)$ and $\left(\xi+t, \xi t^{2}\right)$, whose envelopes are respectively smooth (embedded) and have an order 2 self-tangency. It is easy to see that these two tangential family 

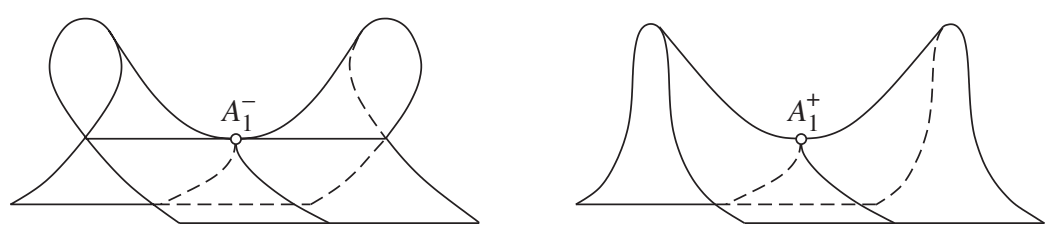

Figure 1. Double Whitney umbrellas.

germs are stable under small tangential deformations. Indeed, the first germ (being a fold) is $\mathcal{A}$-stable. On the other hand, the critical value set of the second germ experiences a beak-to-beak perestroika under any $\mathcal{A}$-miniversal deformation of the germ. Such a perestroika cannot be produced by tangential deformations, so the normal form is stable among tangential family germs. Moreover, one checks that every stable tangential family germ is $\mathcal{A}$-equivalent to one of the above two normal forms.

Consider the projectivized cotangent bundle $P T^{*} \mathbb{R}^{2}$, endowed with the standard contact structure and the standard Legendre fibration $\pi_{\mathrm{L}}: P T^{*} \mathbb{R}^{2} \rightarrow \mathbb{R}^{2}$.

Definition. The Legendrian graph of a tangential family is the surface in $P T^{*} \mathbb{R}^{2}$ formed by the Legendrian lifts of the family curves.

We remark that the envelope of a tangential family is the $\pi_{\mathrm{L}}$-apparent contour of its Legendrian graph.

We say that a Legendrian graph germ is of first type (respectively, second type) if it is generated by a tangential family germ having a singularity I (respectively, II).

Let us recall that a surface germ has a singularity of type $A_{n}^{ \pm}$(respectively, $H_{n}$ ) if it is diffeomorphic to the surface locally parametrized by the map germ $\left(s, t^{2}, t^{3} \pm s^{n+1} t\right)$ (respectively, by $\left(s, s t+t^{3 n-1}, t^{3}\right)$ ); these singularities are simple. The singularities $A_{n}^{+}$ and $A_{n}^{-}$coincide if and only if $n$ is even. The singularities $A_{1}^{ \pm}$, shown in Figure 1, are called double Whitney umbrellas.

Theorem 2.1. The Legendrian graph germs of first type are embedded, while those of second type have generically a double Whitney umbrella singularity $A_{1}^{ \pm}$. The other second-type Legendrian graph germs have $A_{n}^{ \pm}$or $H_{n}$ singularities for $n \geqslant 2$ or $n=\infty$.

In the statement, 'generically' means that the second-type Legendrian graph germs for which the claim does not hold form a (non-connected) codimension 1 submanifold in the manifold formed by the second-type Legendrian graph germs.

Remark. The singularities of map germs from $\mathbb{R}^{2}$ to $\mathbb{R}^{3}$, usually denoted by $B_{n}^{ \pm}$, $C_{n}^{ \pm}, F_{4}$ (see $[4,14]$ ), appear as singularities of Legendrian graph germs generated by non-typical tangential family germs (i.e. which are of neither first nor second type). For example, the Legendrian graphs of $S$-type tangential family germs have $B_{n}^{ \pm}$singularities. Simple tangential family germs are classified in $[\mathbf{8}]$.

A Legendrian graph is stable if, for every small enough tangential deformation of the tangential family generating it, the initial and the deformed graphs are diffeomorphic. A similar definition holds for germs. 
Theorem 2.2. The double Whitney umbrellas $A_{1}^{ \pm}$are, in addition to smooth graphs, the only stable Legendrian graph singularities.

The double Whitney umbrellas are not stable as map germs $\left(\mathbb{R}^{2}, 0\right) \rightarrow\left(\mathbb{R}^{3}, 0\right)$. Indeed, it is a celebrated result of Whitney that the only stable singularity of such a map germ (besides the map germs, whose images have transversal intersections of two or three regular sheets) is a Whitney umbrella (see $[\mathbf{1 4}, \mathbf{1 7}]$ ).

A Legendrian graph singularity $L$ is said to be adjacent to a Legendrian graph singularity $K(L \rightarrow K)$, if every Legendrian graph in $L$ can be deformed into a Legendrian graph in $K$ by an arbitrary small tangential deformation. If $L \rightarrow K \rightarrow K^{\prime}$, the class $L$ is also adjacent to $K^{\prime}$. In this case we omit the arrow $L \rightarrow K^{\prime}$. The adjacencies of the typical Legendrian graph singularities are as follows ( $E$ means embedding):

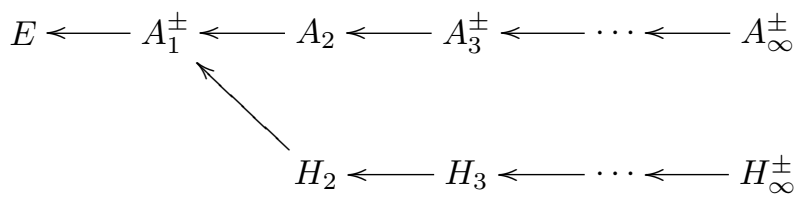

\section{Normal forms of Legendrian graph projections}

In this section we study how Legendrian graphs project into the envelopes of their generating tangential families. In other words, we find normal forms of typical Legendrian graphs with respect to an equivalence relation preserving the fibre structure of $P T^{*} \mathbb{R}^{2}$.

Definition. The projections of two Legendrian graphs $\Lambda_{1}$ and $\Lambda_{2}$ by $\pi_{\mathrm{L}}$ are said to be equivalent if there exists a commutative diagram

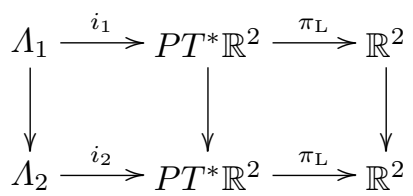

in which the vertical arrows are diffeomorphisms and $i_{1}, i_{2}$ are inclusions.

Such an equivalence is provided by a pair $(\phi, \psi)$, formed by a diffeomorphism $\phi$ between the two Legendrian graphs and a diffeomorphism $\psi: P T^{2} \mathbb{R}^{2} \rightarrow P T^{2} \mathbb{R}^{2}$ fibred over the base $\mathbb{R}^{2}$ (this diffeomorphism is not presumed to be a contactomorphism). A similar definition holds for germs.

Let $\mathcal{A}^{*}$ be the subgroup of $\mathcal{A}:=\operatorname{Diff}\left(\mathbb{R}^{2}, 0\right) \times \operatorname{Diff}\left(\mathbb{R}^{3}, 0\right)$, formed by the pairs $(\phi, \psi)$ such that $\psi$ is fibred with respect to $\pi$. This subgroup inherits the standard action of $\mathcal{A}$ on the maximal ideal $\left(\mathfrak{m}_{s, t}\right)^{3}:(\phi, \psi) \cdot f:=\psi \circ f \circ \phi^{-1}$. Projections of Legendrian graphs are locally equivalent if and only if their local parametrizations are $\mathcal{A}^{*}$-equivalent.

Theorem 3.1. The projection germs of the typical Legendrian graphs are generically equivalent to the projection germs of the surfaces parametrized by the map germs $f$ in 
Table 1. Normal forms of projections of Legendrian graphs.

\begin{tabular}{cccc}
\hline type & singularity & normal form & restrictions \\
\hline I & fold & $\left(s, t^{2}, t\right)$ & $\emptyset$ \\
II & $A_{1}^{ \pm}$ & $\left(s, t^{3}+s t^{2}+a s^{2} t, t^{2}+b t^{3}\right)$ & $a \neq-1,0, a<\frac{1}{3}$ \\
\hline
\end{tabular}
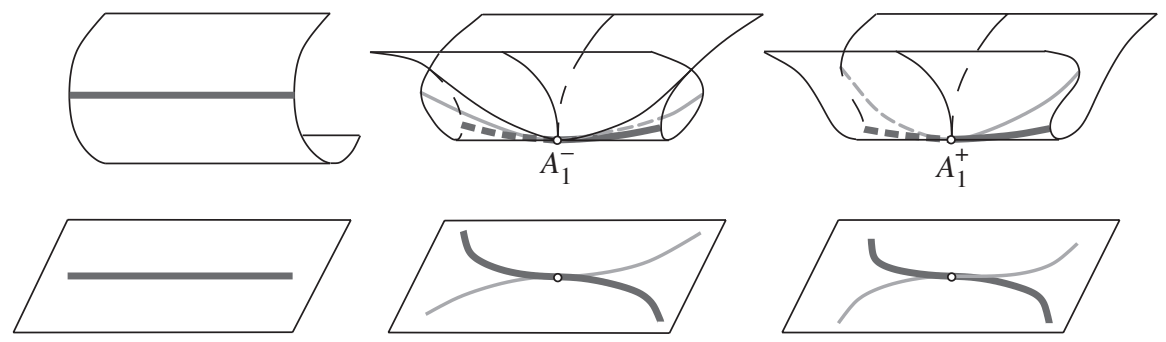

Figure 2. Typical Legendrian graph local projections.

the 3-space $\{x, y, z\}$ by a pencil of lines parallel to the $z$-axis, where $f$ is one of the normal forms listed in Table 1.

Moreover, a Legendrian graph germ of second type, parametrized by the above normal form, has a singularity $A_{1}^{+}$(respectively, $A_{1}^{-}$) if and only if $0<a<\frac{1}{3}$ (respectively, $-1 \neq a<0)$.

Typical Legendrian graph germs are those having only stable singularities. In Theorem 3.1, 'generically' means that the second-type Legendrian graph germs for which the claim does not hold form a non-connected codimension 1 submanifold in the manifold of all the second-type Legendrian graph germs.

Typical local projections of Legendrian graphs are depicted in Figure 2.

Corollary. The fold is the only $\mathcal{A}^{*}$-stable and the only $\mathcal{A}^{*}$-simple singularity of Legendrian graph local projections (with respect to tangential deformations of their generating tangential family germs).

Let $F_{a, b}$ be the $A_{1}^{ \pm}$-normal form in Theorem 3.1 and $z$ its third coordinate $t^{2}+b t^{3}$.

Theorem 3.2. The map germ $F_{a, b}+\left(\mu_{1} z, \lambda t+\mu_{2} z, 0\right)$ is an $\mathcal{A}^{*}$-miniversal deformation of the normal form $F_{a, b}$, provided that $b \neq 0$.

Remark. The above deformation is not the simplest one, but it has the property that the parameters $\mu_{1}, \mu_{2}$ govern the direction of the projection, leaving unchanged the Legendrian graph, while the parameter $\lambda$ deforms the graph, without changing the projection. In particular, the deformation restricted to $\mu_{1}=\mu_{2}=0$ provides an $\mathcal{A}$ miniversal deformation of $F_{a, b}$.

The second-order self-tangency of the envelope of a second-type tangential family germ is not stable under non-tangential deformations (see $[\mathbf{7}, \mathbf{1 6}]$ ). Under such a deformation, the envelope experiences a beak-to-beak perestroika, which may be interpreted as the 


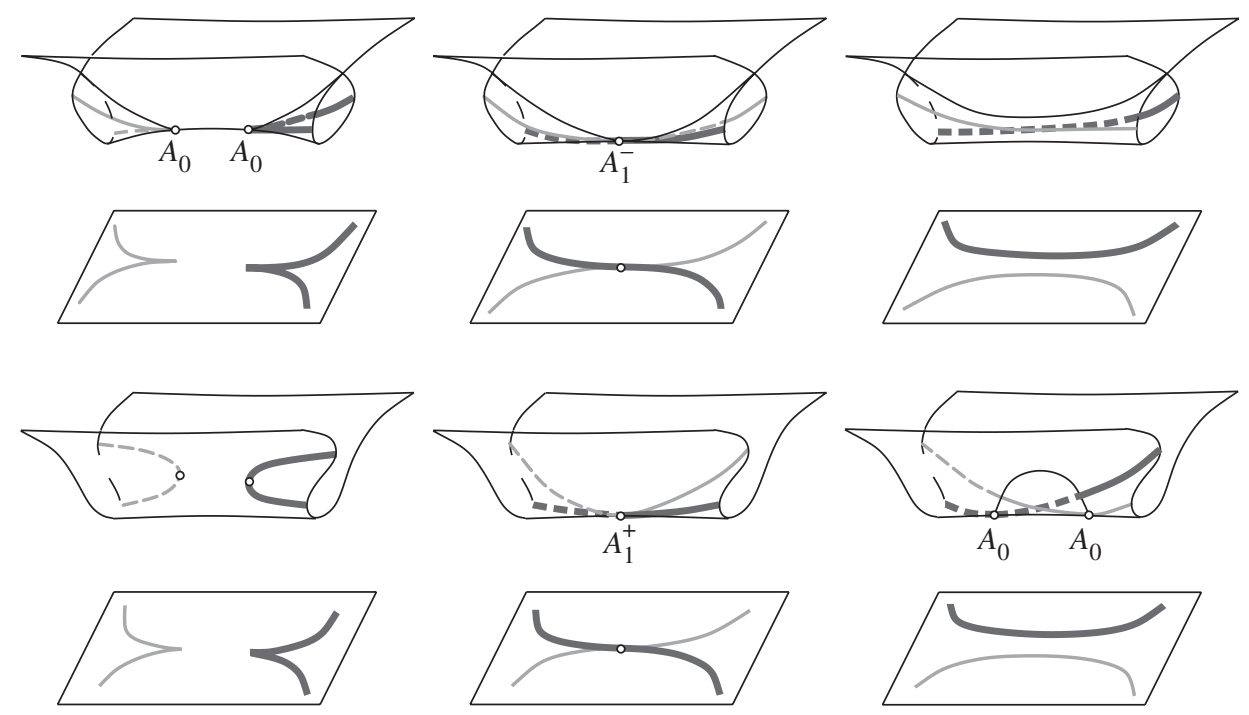

Figure 3. Legendrian beak-to-beak perestroikas.

apparent contour in the plane of the perestroika of the Legendrian graph, as shown in Figure 3. We call it Legendrian beak-to-beak perestroika. Actually, there are two such perestroikas, according to the sign of $A_{1}^{ \pm}$. Figure 3 was obtained while investigating the critical sets of the $\mathcal{A}$-miniversal deformation $F_{a, b}+(0, \lambda t, 0)$ of the projection normal form $F_{a, b}$, which leaves the direction of the projection unchanged.

\section{Proof of Theorems 2.1 and 2.2}

We start constructing explicit parametrizations of Legendrian graph germs.

Lemma 4.1. Every local parametrization of a Legendrian graph is $\mathcal{A}^{*}$-equivalent to a map germ of the form

$$
\left(s, k_{0} t^{2}+\left(\alpha-k_{1}\right) t^{3}+k_{1} s t^{2}+\delta(3), 2 k_{0} t+\left(3 \alpha-2 k_{1}\right) t^{2}+2 k_{1} s t+\delta(2)\right),
$$

where $\delta(n)$ denotes any function of $s, t$ with zero $n$-jet at the origin. Moreover, the Legendrian graph germ is of first type (respectively, of second type) if and only if $k_{0} \neq 0$ (respectively, $k_{0}=0$ and $k_{1} \neq 0, \alpha$ ).

Proof. Consider a tangential family germ at the origin. Up to a coordinate change, preserving the $\mathcal{A}^{*}$-singularity of the graph, we may assume that the family support is locally the $x$-axis. For every $x$ small enough, denote by $K(x)$ the curvature at $(x, 0)$ of the corresponding family curve. Now, for $x \rightarrow 0$, let $k_{0}+k_{1} x+o(x)$ and $k_{0} x^{2}+\alpha x^{3}+o\left(x^{3}\right)$ be the expansions of $\frac{1}{2} K(x)$ and of the function whose graph (near the origin) is the curve associated with $(0,0)$.

Then, one easily verifies that the Legendrian graph of such a tangential family is parametrized by $\left(\xi+t, u(\xi, t)+\delta(3), \partial_{t} u(\xi, t)+\delta(2)\right)$, where $u(\xi, t):=k_{0} t^{2}+\alpha t^{3}+k_{1} \xi t^{2}$. This germ can be brought to the required form by setting $s=\xi+t$. 
Finally, we proved in [7] that a tangential family germ is of first type (respectively, of second type) if and only if $k_{0} \neq 0$ (respectively, $k_{0}=0$ and $k_{1} \neq 0, \alpha$ ).

We can now prove Theorems 2.1 and 2.2 .

Proof of Theorem 2.1. If $k_{0} \neq 0$, then the 1 -jet of $(4.1)$ is $\mathcal{A}$-equivalent to $(s, 0, t)$, which is $\mathcal{A}$-sufficient. Therefore, Legendrian graph germs of first type are embedded.

We now consider second-type Legendrian graph germs (so from now on $k_{0}=0$ ). First, assume that $k_{1}$ is different from the four values $0, \alpha, \frac{3}{2} \alpha$ and $3 \alpha$. Then (4.1) is $\mathcal{A}$-equivalent to $\left(s, t^{3} \pm t s^{2}, t^{2}\right)$, where ' \pm ' is the sign of $\left(k_{1}-3 \alpha\right)\left(\alpha-k_{1}\right) / k_{1}^{2}$. Indeed, the 3 -jet of (4.1) is $\mathcal{A}$-equivalent to $\left(s, t^{3} \pm s^{2} t, t^{2}\right)$, which is $\mathcal{A}$-sufficient (see [14, Theorem 1:2]).

Hence, the Legendrian graph germs of second type have an $A_{1}^{ \pm}$singularity whenever $k_{1} \neq \frac{3}{2} \alpha, 3 \alpha$. Let us denote by II the manifold formed by all the second-type Legendrian graph local parametrizations. The remaining second-type graphs belong to the union of the two submanifolds of II, defined by $2 k_{1}=3 \alpha$ and $3 \alpha=k_{1}$ (dropping the intersection $\alpha=k_{1}=0$, whose elements are not of second type). It remains to consider the germs belonging to these two submanifolds.

If $3 \alpha=2 k_{1} \neq 0$, the 3 -jet of $(4.1)$ is $\mathcal{A}$-equivalent to $\left(s, t^{3}, s t\right)$. Then, Mond's classification $[\mathbf{1 4}, \S 4.2 .1]$ implies that the map germs (4.1), except those belonging to an infinite codimension submanifold of $\hat{\mathrm{II}}$, are $\mathcal{A}$-equivalent to $\left(s, t^{3}, s t+t^{3 n-1}\right)$ for some $n \geqslant 2$. On the other hand, when $k_{1}=3 \alpha \neq 0$, the 2 -jet of (4.1) is $\mathcal{A}$-equivalent to $\left(s, 0, t^{2}\right)$; Mond's classification $[\mathbf{1 4}, \S 4.1]$ implies that the map germs of the form (4.1), except those belonging to an infinite codimension submanifold of II, are $\mathcal{A}$-equivalent to $\left(s, t^{3} \pm s^{n+1} t, t^{2}\right)$ for $n \geqslant 2$.

Proof of Theorem 2.2. We first show that $A_{1}^{ \pm}$singularities are stable. It is well known that $\left(s, t^{3} \pm s t^{2}+\lambda t, t^{2}\right)$ is a miniversal deformation of $A_{1}^{ \pm}$(the singularity being of codimension 1 (see $[\mathbf{1 4}])$ ). No tangential deformation can be equivalent to it, since it induces a beak-to-beak perestroika on the corresponding envelope. Therefore, every tangential deformation of the singularity is trivial, due to the envelope stability.

On the other hand, the non-typical Legendrian graphs of second type are not stable, due to the adjacencies $A_{n+1}^{ \pm} \rightarrow A_{n}^{ \pm}, A_{\infty} \rightarrow A_{n}^{ \pm}, H_{n+1}^{ \pm} \rightarrow H_{n}^{ \pm}$and $H_{\infty} \rightarrow H_{n}^{ \pm}$(these adjacencies are obtained by small tangential deformations).

Finally, as proven in [7], a tangential family germ neither of first nor second type can be deformed into a second-type tangential family germ via an arbitrary small tangential deformation. Hence, its Legendrian graph singularity is adjacent to $A_{1}^{ \pm}$.

\section{Proof of Theorems 3.1 and 3.2}

In this section we prove Theorems 3.1 and 3.2 (for details about computations we refer to $[\mathbf{6}])$. In order to follow the usual scheme for this reduction, we recall that a finite determinacy theorem for $\mathcal{A}^{*}$-equivalence relation has been proven by Goryunov in $[\mathbf{1 1}]$; this result follows also from Damon's theory about nice geometric subgroups of $\mathcal{A}$ (see, for example, $[\mathbf{9}])$. 
A map germ $f \in\left(\mathfrak{m}_{s, t}\right)^{3}$ defines, by $f^{*} g:=g \circ f$, a homomorphism from the ring $\mathcal{E}_{x, y, z}$ of the function germs in the target to the ring $\mathcal{E}_{s, t}$ of the function germs in the source. Hence, every $\mathcal{E}_{s, t}$-module has the structure of $\mathcal{E}_{x, y, z}$-module via this homomorphism. We define the extended tangent space of $f$ as usual by

$$
T_{e} \mathcal{A}^{*}(f):=\left\langle\partial_{s} f, \partial_{t} f\right\rangle_{\mathcal{E}_{s, t}}+f^{*}\left(\mathcal{E}_{x, y}\right) \times f^{*}\left(\mathcal{E}_{x, y}\right) \times f^{*}\left(\mathcal{E}_{x, y, z}\right) .
$$

Note that $T_{e} \mathcal{A}^{*}(f)$ is an $\mathcal{E}_{x, y}$-module, being in general neither an $\mathcal{E}_{s, t}$-module nor an $\mathcal{E}_{x, y, z}$-module. The reduced tangent space $T_{r} \mathcal{A}^{*}(f)$ of $f$ is, by definition, the $\mathcal{E}_{x, y^{-}}$ submodule of $T_{e} \mathcal{A}^{*}(f)$ defined by $\mathfrak{g}_{+}+\mathcal{M}^{*}$, where $\mathfrak{g}_{+}$is the space of all the vector field germs having positive order (see $[\mathbf{3}]$ for definitions) and $\mathcal{M}^{*}$ is the following $\mathcal{E}_{x, y}$-module:

$$
f^{*}\left(\mathfrak{m}_{x, y}^{2} \oplus\langle y\rangle_{\mathbb{R}}\right) \times f^{*}\left(\mathfrak{m}_{x, y}^{2} \oplus\langle x\rangle_{\mathbb{R}}\right) \times f^{*}\left(\mathfrak{m}_{x, y, z}^{2} \oplus\langle x, y\rangle_{\mathbb{R}}\right) .
$$

The main tool in the proof of Theorems 3.1 and 3.2 is the following easily determined fact.

Lemma 5.1. Let $f \in\left(\mathfrak{m}_{s, t}\right)^{3}$ be a map germ and let $R$ be a triple of homogeneous polynomials of degree $p, q$ and $r$, such that $R \in T_{r} \mathcal{A}^{*}(f)+\left(\mathfrak{m}_{s, t}^{p+1} \times \mathfrak{m}_{s, t}^{q+1} \times \mathfrak{m}_{s, t}^{r+1}\right)$. Then the $(p, q, r)$-jets of $f$ and $f+R$ are $\mathcal{A}^{*}$-equivalent.

We can now start the proof of Theorem 3.1.

Proof of Theorem 3.1. First we consider Legendrian graphs of first-type tangential family germs $\left(k_{0} \neq 0\right)$. Then the 2 -jet of $(4.1)$ is $\mathcal{A}^{*}$-equivalent to $\left(s, t^{2}, t\right)$, which is $\mathcal{A}^{*}$-sufficient, since its reduced tangent space contains $\mathfrak{m}_{s, t}^{2} \times \mathfrak{m}_{s, t}^{3} \times \mathfrak{m}_{s, t}^{2}$ (Lemma 5.1).

Now we consider Legendrian graphs of second-type tangential family germs $\left(k_{0}=0\right)$. In this case, every map germ (4.1) is $\mathcal{A}^{*}$-equivalent to $\left(s, t^{3}+s t^{2}+a s^{2} t+\delta(3), t^{2}+\delta(2)\right)$, where $a:=\left(\alpha-k_{1}\right)\left(k_{1}-3 \alpha\right) / k_{1}^{2}$. We remark that $a<\frac{1}{3}$; indeed, we have $1-3 a=$ $\left(3 \alpha-2 k_{1}\right)^{2} / k_{1}^{2}>0$, since $3 \alpha \neq 2 k_{1}$. Actually, a further computation shows that its $(2,4,3)$-jet is $\mathcal{A}^{*}$-equivalent to $F_{a, b}$, for a suitable $b \in \mathbb{R}\left(F_{a, b}\right.$ is the normal form defined in $\S 3)$. Hence, the statement follows from Lemma 5.1 and the next inclusion, which holds for $a \neq-1,0, \frac{1}{3}$ :

$$
\mathfrak{m}_{s, t}^{3} \times \mathfrak{m}_{s, t}^{5} \times \mathfrak{m}_{s, t}^{4} \subset T_{r} \mathcal{A}^{*}\left(F_{a, b}\right) .
$$

When the Legendrian graph has an $A_{1}^{ \pm}$singularity, the conditions $a \neq 0, \frac{1}{3}$ are automatically fulfilled. On the other hand, $a \neq-1$ is a new condition, equivalent to $\alpha=0$, giving rise to the submanifold for which Theorem 3.1 does not hold.

Proof of Theorem 3.2. Since $\left\langle s, t^{3}+s t^{2}+a s^{2} t\right\rangle_{\mathcal{E}_{\xi, t}}=\left\langle s, t^{3}\right\rangle_{\mathcal{E}_{s, t}}$ and

$$
\left\langle s, t^{3}+s t^{2}+a s^{2} t, t^{2}+b t^{3}\right\rangle_{\mathcal{E}_{\xi, t}}=\left\langle s, t^{2}\right\rangle_{\mathcal{E}_{s, t}},
$$

the well-known preparation theorem of Mather and Malgrange (see, for example, $[\mathbf{3}]$ ) implies that $\mathcal{E}_{s, t}$ is generated by $\left\{t, t^{2}\right\}$ as an $\mathcal{E}_{x, y}$-module and by $t$ as an $\mathcal{E}_{x, y, z}$-module. Hence, we have

$$
\mathcal{E}_{s, t}^{3}=F_{a, b}^{*}\left(\mathcal{E}_{x, y}\right) \cdot\left\{\left(\begin{array}{l}
t \\
0 \\
0
\end{array}\right),\left(\begin{array}{l}
0 \\
t \\
0
\end{array}\right),\left(\begin{array}{c}
t^{2} \\
0 \\
0
\end{array}\right),\left(\begin{array}{c}
0 \\
t^{2} \\
0
\end{array}\right)\right\}+F_{a, b}^{*}\left(\mathcal{E}_{x, y, z}\right) \cdot\left\{\left(\begin{array}{l}
0 \\
0 \\
t
\end{array}\right)\right\} .
$$


Setting (as in $\S 3) z=t^{2}+b t^{3}$, for $b \neq 0$ we obtain the equality

$$
\mathcal{E}_{s, t}^{3}=T_{e} \mathcal{A}^{*}\left(F_{a, b}\right) \oplus \mathbb{R} \cdot\left\{\left(\begin{array}{l}
0 \\
t \\
0
\end{array}\right),\left(\begin{array}{l}
z \\
0 \\
0
\end{array}\right),\left(\begin{array}{l}
0 \\
z \\
0
\end{array}\right)\right\} .
$$

This proves the theorem.

Acknowledgements. This paper contains some of the results of my $\mathrm{PhD}$ thesis $[6]$. I express my deep gratitude to my advisor, V. I. Arnol'd. I also thank the referee of this paper for suggesting references $[\mathbf{1 3}]$ and $[\mathbf{1 5}]$.

\section{References}

1. V. I. ARnol'D, On the envelope theory, Usp. Mat. Nauk 31(3) (1976), 248-249 (in Russian).

2. V. I. ARnol'D, Wave front evolution and equivariant Morse lemma, Commun. Pure Appl. Math. 29(6) (1976), 557-582.

3. V. I. Arnol'd, S. M. Guseřn-Zade and A. N. Varchenko, Singularities of differentiable maps, vol. I (Birkhäuser, Basel, 1985).

4. V. I. Arnol'd, V. V. Goryunov, O. V. Lyashko and V. A. Vasiliev, Singularity theory II, Dynamical Systems VIII, Encyclopaedia of Mathematical Sciences, vol. 39 (Springer, 1993).

5. J. W. Bruce and P. J. Giblin, Projections of surfaces with boundary, Proc. Lond. Math. Soc. (3) 60 (1990), 392-416.

6. G. Capitanio, Familles tangentielles et solutions de minimax pour l'équation de Hamilton-Jacobi, PhD thesis (in English), University of Paris VII, 2004 (available at www.institut.math.jussieu.fr/theses/2004/capitanio/).

7. G. Capitanio, Stable tangential family germs and singularities of their envelopes, $C . R$. Acad. Sci. Paris Sér. I 341 (2005), 503-508.

8. G. Capitanio, Simple tangential families and perestroikas of their envelopes, Bull. Soc. Math. France, in press.

9. J. Damon, The unfolding and determinacy theorems for subgroups of $\mathcal{A}$ and $\mathcal{K}$, in Singularities, Part 1, Arcata, CA, 1981, pp. 233-254, Proceedings of Symposia of Pure Mathematics, vol. 40 (American Mathematical Society, Providence, RI, 1983).

10. A. B. Givental, Singular Lagrangian varieties and their Lagrangian mappings, J. Sov. Math. 33 (1990), 3246-3278.

11. V. V. Goryunov, Singularities of projections of complete intersections, J. Sov. Math. $\mathbf{2 7}$ (1984), 2785-2811.

12. V. V. GoRYunov, Projections of generic surfaces with boundaries, in Theory of singularities and its applications, pp. 157-200, Advances in Soviet Mathematics, vol. 1 (American Mathematical Society, Providence, RI, 1990).

13. S. Izumiya and Y. Kurokawa, Holomorphic systems of Clairaut type, Diff. Geom. Applic. 5 (1995), 219-235.

14. D. Mond, On the classification of germs of maps from $\mathbb{R}^{2}$ to $\mathbb{R}^{3}$, Proc. Lond. Math. Soc. (3) 50 (1985), 333-369.

15. M. TAKAhashi, Bifurcations of ordinary differential equations of Clairaut type, J. Diff. Eqns 190 (2003), 579-599.

16. R. Тном, Sur la théorie des enveloppes, J. Math. Pures Appl. 41(9) (1962), 177-192.

17. H. Whitney, Tangents to an analytic variety, Ann. Math. (2) 81 (1965), 496-549. 